\title{
CFTR targeted therapies: recent advances in cystic fibrosis and possibilities in other diseases of the airways
}

\author{
Sheylan D. Patel ${ }^{1,3}$, Taylor R. Bono ${ }^{1,2,3}$, Steven M. Rowe $\mathbb{1}^{1,2}$ and \\ George M. Solomon ${ }^{1,2}$
}

\author{
Number 4 in the Series "Controversies in bronchiectasis" \\ Edited by James Chalmers and Michal Shteinberg
}

Affiliations: ${ }^{1}$ Dept of Medicine, University of Alabama at Birmingham, Birmingham, AL, USA. ${ }^{2}$ The Gregory Fleming James Cystic Fibrosis Research Center, University of Alabama at Birmingham, Birmingham, $A L$, USA. ${ }^{3}$ Both authors contributed equally.

Correspondence: Steven M. Rowe, MD MSPH, MCLM 7061918 University Blvd, Birmingham, AL 35294-0006, USA. E-mail: smroweduab.edu

@ERSpublications

CFTR dysfunction may be part of the pathophysiology of many diseases of the airways. Exploration of mechanisms of dysfunction and options for CFTR-directed therapies are examined in this article. http://bit.ly/33o6nDu

Cite this article as: Patel SD, Bono TR, Rowe SM, et al. CFTR targeted therapies: recent advances in cystic fibrosis and possibilities in other diseases of the airways. Eur Respir Rev 2020; 29: 190068 [https://doi.org/ 10.1183/16000617.0068-2019].

ABSTRACT Cystic fibrosis transmembrane conductance regulator (CFTR) is an ion transporter that regulates mucus hydration, viscosity and acidity of the airway epithelial surface. Genetic defects in CFTR impair regulation of mucus homeostasis, causing severe defects of mucociliary clearance as seen in cystic fibrosis. Recent work has established that CFTR dysfunction can be acquired in chronic obstructive pulmonary disease (COPD) and may also contribute to other diseases that share clinical features of cystic fibrosis, such as asthma, allergic bronchopulmonary aspergillosis and bronchiectasis. Protean causes of CFTR dysfunction have been identified including cigarette smoke exposure, toxic metals and downstream effects of neutrophil activation pathways. Recently, CFTR modulators, small molecule agents that potentiate CFTR or restore diminished protein levels at the cell surface, have been successfully developed for various CFTR gene defects, prompting interest in their use to treat diseases of acquired dysfunction. The spectrum of CFTR dysfunction, strategies for CFTR modulation, and candidate diseases for CFTR modulation beyond cystic fibrosis will be reviewed in this manuscript.

Previous articles in this series: No. 1: Amati F, Simonetta E, Gramegna A, et al. The biology of pulmonary exacerbations in bronchiectasis. Eur Respir Rev 2019; 28: 190055. No. 2: Shteinberg M, Flume PA, Chalmers JD. Is bronchiectasis really a disease? Eur Respir Rev 2019; 28: 190051. No. 3: Tiddens HAWM, Meerburg JJ, van der Eerden MM. The radiological diagnosis of bronchiectasis: what's in a name? Eur Respir Rev 2019; 28: 190120.

Provenance: Commissioned article, peer reviewed.

Received: 13 June 2019 | Accepted after revision: 9 March 2020

Copyright $\odot$ ERS 2020. This article is open access and distributed under the terms of the Creative Commons Attribution Non-Commercial Licence 4.0. 


\section{Cystic fibrosis background}

Cystic fibrosis is an autosomal recessive genetic disorder with an estimated incidence of about one in 3000 births [1] in a North American (USA) cohort, and is the most common monogenetic disease of people of Caucasian descent. Cystic fibrosis is caused by a mutation in the cystic fibrosis transmembrane conductance regulator (CFTR) gene, which leads to various defects in the CFTR protein. In the lung, CFTR dysfunction is associated with altered mucus clearance, recurrent respiratory infections, and ultimately respiratory failure characteristic of cystic fibrosis [1]. CFTR is also expressed beyond the respiratory tract, which commonly presents as pancreatic insufficiency, intestinal obstruction and male infertility in cystic fibrosis.

Cystic fibrosis shares many features with other airway disorders including altered mucus properties and clearance [2]. As such, these disorders may lie along a continuum of severity. Due to the shared pathological findings of impaired mucociliary clearance, altered mucus properties and goblet cell hyperplasia, common mechanisms of disease have been proposed, including CFTR dysfunction, from genetic and non-genetic causes [3].

\section{CFTR physiology and contribution to disease}

The sinopulmonary tract is continuously exposed to the outside environment. While the lungs and sinuses have several innate defence mechanisms, of particular importance is the mucociliary clearance apparatus. The airway mucus layer, a component of the airway surface liquid (ASL), functions as a physical barrier and a complement to innate immunity by trapping bacteria and harmful particles and is then swept by cilia up and out of the trachea to be expectorated or swallowed [4]. Normal CFTR function, via transport of chloride anions into the luminal space, regulates the hydration status of mucus, which is directly associated with mucociliary transport (MCT) efficiency [5]. In addition, CFTR-mediated transport of bicarbonate is co-stimulated by mucus release into the airways $[6,7]$, regulates ASL pH necessary for normal lactoferrin function and other defensins [8], and aids in mucin post-translational unpacking to impart normal rheologic properties, including viscosity and transportability. CFTR function also imparts regulation of other ion channels including the epithelial sodium channel (ENaC) [9]. Dysfunction of CFTR is accompanied by unopposed $\mathrm{ENaC}$ function, contributing to ASL depletion and mucus dehydration [10-12].

Patients in whom CFTR is dysfunctional, either by genetic or acquired causes, may exhibit mucus that is more viscous, abnormally adherent and more acidic. These changes also inhibit ciliary function [13, 14]. This pathological process translates to decreased mucociliary clearance and increased mucus stasis which, together with altered host defence, predisposes to chronic infection [15] and dysregulated inflammation [16].

\section{Genetic and acquired causes of CFTR dysfunction}

More than 1900 CFTR variants have been identified, of which nearly 400 have been confirmed to be disease-causing $[17,18]$. The most common CFTR mutation is the deletion of bases coding for the amino acid phenylalanine at the 508th position (F508del) $[18,19]$. This mutation accounts for approximately $70-80 \%$ of all cystic fibrosis-causing mutations, and just under $50 \%$ of individuals with cystic fibrosis are homozygous for F508del [20]. Broadly, CFTR mutations have been organised into a class scheme that groups them by the principal cellular mechanism by which they cause disease: abnormalities in protein production, protein processing and trafficking, channel gating, channel conductance, protein quantity or protein stability $[18,21]$. While a useful construct to understanding the pathobiology of CFTR mutations, this system has limitations that are important to highlight. Foremost, while mutations are classified based on the predominant defect, an individual mutation may affect several processes in concert, such as protein processing and trafficking and channel function [22]. For example, F508del primarily manifests as a misfolded protein, most of which undergoes degradation through post-translational processing. The small amount of mature protein that reaches the cell surface exhibits defective channel gating as well as cell surface instability, leading to premature recycling [22-24]. As such, the classification system can be imprecise and may oversimplify the extent to which genotype affects protein expression and function.

In addition to molecular defects in the CFTR protein itself, the CFTR gene can also be affected by modifier genes and environmental factors, further contributing to the variability in CFTR expression and anion transport [25]. Ultimately, while cystic fibrosis phenotype is largely determined by genotype, the genotypephenotype relationship is complex, influenced by multiple factors that have not been completely elucidated.

Recently, non-genetic CFTR dysfunction has garnered interest. The first descriptions linked cigarette smoke to abnormal chloride transport prior to identification of the CFTR gene [26], and have since been found to include CFTR dysfunction in healthy smokers and smokers with chronic obstructive pulmonary disease (COPD) [26-30]. CFTR dysfunction has been observed in both the upper and lower airways in patients with chronic bronchitis [27,31]. These patients share sinopulmonary phenotypic features that are similar, albeit less severe, to patients with classic (genetic) cystic fibrosis and exhibit sweat gland 
abnormalities characteristic of mild CFTR deficits [32-34]. Aside from the direct effects of cigarette smoking, neutrophil-driven inflammation can impair CFTR function due to the effects of neutrophil elastase and neutrophil-derived protease degradation products [35]. Ciliary paralysis leading to loss of purinergic regulation of epithelial ion transport may also indirectly impair CFTR activity in diseases of primary or secondary ciliary dysfunction $[36,37]$. Each of these has the potential to confer acquired, non-genetic CFTR dysfunction, which can contribute to a specific clinical sub-phenotype characterised by abnormalities of mucus and host defence.

\section{CFTR-directed treatment strategies}

Early treatment strategies for cystic fibrosis focussed on improving mucus clearance, managing chronic infections, and improving nutrition $[38,39]$. Nebulised therapies in combination with chest physiotherapy improve mucus mobilisation, augmenting mucin clearance. Inhaled antibiotics are utilised to control chronic infections and attempt to prevent infection-associated exacerbations of airway disease. In addition, anti-inflammatory medications aimed at controlling aspects of unchecked inflammation have been identified but are not widely adopted [40]. These symptomatic therapies do not directly target the intrinsic defect and as a result, only focus on the consequences of impaired mucociliary clearance [41].

More recently, a new class of therapeutics called CFTR modulators, small molecule agents that potentiate CFTR or restore diminished protein levels at the cell surface, have been developed. These therapeutics directly address the functional protein defects that cause cystic fibrosis. There is the potential that this new class of therapeutics may also have a role in treating non-genetic CFTR dysfunction given the increasing evidence that acquired CFTR abnormalities contribute to other pulmonary disorders.

Developing these modulators was made possible by a high throughput screening (HTS) combined with cell models (human bronchial epithelial (HBE)) that accurately replicate the pathophysiological defect. Rather than designing molecules for a specific target, large repositories of diverse chemical compounds were screened to assess for a response against multiple CFTR mutations identifying potential therapeutics. Our group has previously reviewed this process in more depth [21]. The HTS process identified several possible potentiators, the first class of modulators developed, ultimately resulting in the development of ivacaftor based on several factors such efficacy and pharmacokinetic profile [42].

CFTR potentiators improve chloride conduction by increasing open probability of the CFTR channel. The potentiator ivacaftor led to robust improvements in several important clinical metrics in patients with the G551D gating mutation including forced expiratory volume in $1 \mathrm{~s}\left(\mathrm{FEV}_{1}\right)$, sweat chloride, exacerbation rates and body mass index (BMI) [43]. Average sweat chloride levels improved from $100 \mathrm{mmol} \cdot \mathrm{L}^{-1}$ to $47.8 \mathrm{mmol} \cdot \mathrm{L}^{-1}$, where $60.0 \mathrm{mmol} \cdot \mathrm{L}^{-1}$ and above is traditionally diagnostic for cystic fibrosis [43]. These improvements with ivacaftor served as a benchmark for the potential efficacy of highly effective CFTR modulators and how these therapeutics are developed and approved for cystic fibrosis.

Recent progress in the development of cystic fibrosis therapies has been bolstered by cell models that strongly predict results in clinical trials. In vitro testing supported ivacaftor's benefit with several other non-G551D gating mutations [44] which was subsequently confirmed in clinical trials resulting in an expanded approval for ivacaftor for non-G551D mutations $[45,46]$. Based on this experience, in 2017, the US Food and Drug Administration (FDA) made the novel decision to allow the approval of modulators based on in vitro testing $[46,47]$. This process employing in vitro outcome measures also overcomes the challenge of studying rare mutations where large clinical trials are not practical or feasible.

Ivacaftor as a single agent was not predicted to work in F508del mutations because the predominant effect of the mutation is a misfolded CFTR protein that is degraded before it reaches the cell surface. To restore function for this class of mutations, treatment is required to correct protein folding and trafficking, and prevent post-translational degradation, improving cell surface expression. These agents, called correctors, increase cell surface expression and stability; however, function is not normalised, as F508del exhibits a concomitant gating defect that reduces its ion transport activity [23]. This gating defect is responsive to ivacaftor [42]. Lumacaftor was the first CFTR corrector for which efficacy was demonstrated, when combined with ivacaftor, for F508del-homozygous individuals [23, 42, 48]. Definitive studies showed a modest improvement in $\mathrm{FEV}_{1}$, BMI, and exacerbation frequency $[49,50]$ compared with the effect seen with ivacaftor with G551D [43].

An alternative CFTR corrector, tezacaftor, was developed with advantageous pharmacological properties but a similar mechanism of action. When used in combination with ivacaftor, tezacaftor improved spirometry, exacerbation risk and sweat chloride, displaying similar levels of efficacy as lumacaftor but with fewer respiratory-related adverse effects associated with off-target effects [51]. Additionally, tezacaftor/ ivacaftor was effective in both F508del homozygotes and F508del heterozygotes with a concomitant residual function mutation [52]. Figure 1 summarises the response of various CFTR modulators in clinical trials as measured by change in sweat chloride concentration from baseline. 


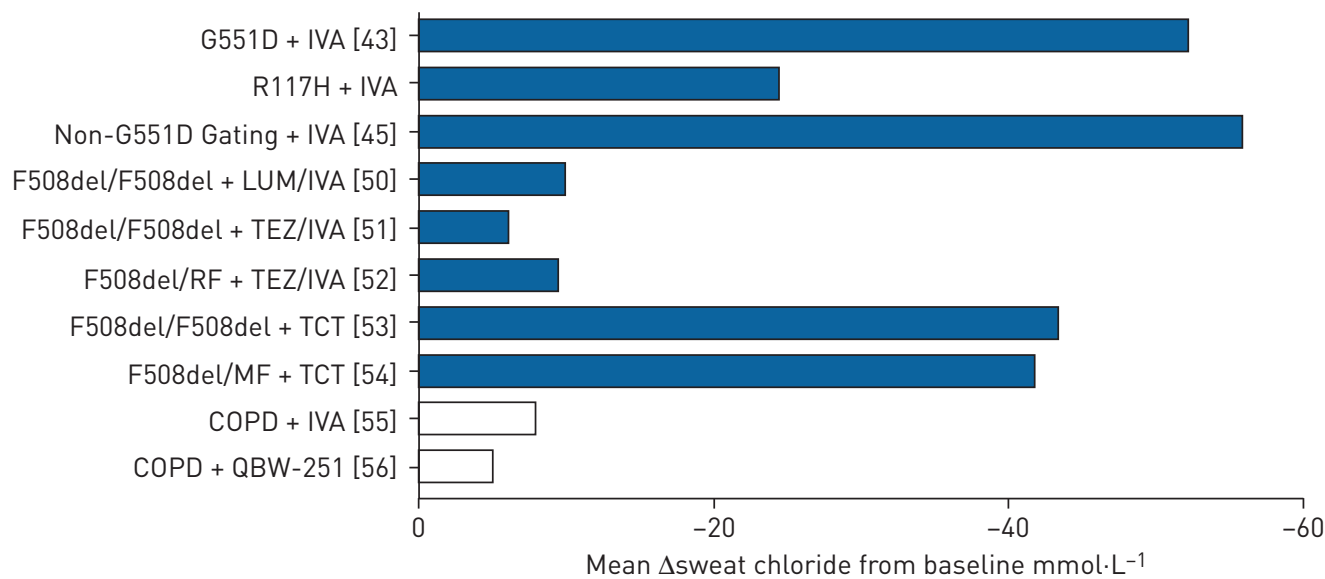

FIGURE 1 Summary of mean sweat chloride response to cystic fibrosis transmembrane conductance regulator modulators in cystic fibrosis and COPD by disease state in major clinical trials. IVA: ivacaftor; LUM: lumacaftor; TEZ: tezacaftor; TCT: triple combination of elexacaftor (VX-445)/tezacaftor/ivacaftor.

While tezacaftor/ivacaftor improved patient outcomes [51], benefit was modest when compared with the effects seen in early trials of ivacaftor with G551D [43]. Sweat chloride levels with tezacaftor/ivacaftor decreased by $\sim 15 \mathrm{mmol} \cdot \mathrm{L}^{-1}$ compared with $\sim 50 \mathrm{mmol} \cdot \mathrm{L}^{-1}$ seen with ivacaftor in G551D [43, 51] (figure 1). Approaches evaluating three-drug combinations consisting of mechanistically distinct correctors on top of tezacaftor/ivacaftor have therefore been explored. In phase 3 studies in patients with one F508del allele, a triple combination approach of the next-generation corrector elexacaftor with tezacaftor/ivacaftor elicited marked improvements in $\mathrm{FEV}_{1}$, pulmonary exacerbations and respiratory symptoms compared with placebo [54]. Benefits with the three-drug combination were seen in F508del homozygotes [53] as well as heterozygotes with a minimal function mutation [54]. These results, together with a favourable safety profile, led to the recent FDA approval of elexacaftor/tezacaftor/ivacaftor for people with cystic fibrosis with at least one F508del allele, covering nearly $90 \%$ of the cystc fibrosis population genetically.

\section{CFTR dysfunction in non-CF diseases}

Impaired CFTR function in the absence of genetic mutation is increasingly recognised outside of cystic fibrosis. Diminished chloride conductance after smoke exposure [26] was among the first evidence of what is now known to be an acquired defect of the CFTR channel. More recently, several mechanisms have been shown to impair CFTR function, including toxins in cigarette smoke through reduced channel gating [57, 58], CFTR degradation through $\mathrm{Ca}^{2+}$ induced internalisation [35], acute influenza [59] and neutrophil degradation pathways. The clinical and therapeutic significance of each of these pathways requires further investigation. Herein, we will update recent advances in understanding of the landscape of acquired CFTR dysfunction, and discuss potential roles of CFTR modulators in diseases of the respiratory tract beyond cystic fibrosis.

\section{CFTR dysfunction in COPD and smoking-related disease}

First demonstrated as early as the 1980s prior to the identification of CFTR, smoke exposure was shown to reduce chloride transport in canine airways through what is now known to be an acquired defect of CFTR [26]. CFTR dysfunction was subsequently confirmed in cell models and human subjects via nasal potential difference (NPD) measurements $[28,31]$. These effects appear to be partially, but not completely, reversible after cessation of smoke exposure, supporting a causal relationship between cigarette smoke and CFTR dysfunction [60].

Similar evidence of CFTR dysfunction has been shown in COPD. Active smokers with COPD had reduced CFTR function and expression in the upper respiratory tract [31], whereas non-smokers, defined as individuals who abstained from smoking for at least a year and had a negative urine cotinine test, did not have CFTR dysfunction [27, 31]. Similar results were found in the lower respiratory tract of active smokers with and without COPD as measured by lower airway potential difference (LAPD) and was accompanied by reductions in CFTR surface protein expression as assessed by Western blot [27]. Regardless of the presence of COPD, patients without continued smoke exposure had less severe deficits of CFTR function in the lung $[27,31]$. In addition to this finding, the positive correlation between CFTR functional impairment, observed in both nasal and lung potential difference, and symptoms of chronic bronchitis [27, 31] further implicate a causal relationship between smoke exposure and CFTR dysfunction and suggest a potential therapeutic pathway for patients with a chronic bronchitis COPD phenotype. 
Smoke-induced CFTR dysfunction is not limited to the respiratory tract and can be detected at anatomic sites remote to the exposure. Compared with never smokers, smokers exhibited noticeable differences in sweat chloride concentration [32,33] and intestinal current measurements [33] that corresponded to $\sim 40 \%$ and $\sim 60 \%$ reductions in CFTR function, respectively. In comparison, obligate carriers demonstrate $\sim 50 \%$ of wild-type CFTR activity, and CFTR activity is $<10 \%$ of wild-type in overt cystic fibrosis disease in the sweat gland $[55,61]$. Similar CFTR impairments have been observed in mice with smoke exposure limited only to the respiratory tract [33]; the degree of impairment was directly related to the duration and intensity of exposure, with the intestines showing a delayed and blunted response compared with the respiratory tract, consistent with a less intense and non-localised systemic exposure [33]. Additionally, incubating human bronchial epithelial cells from healthy volunteers with plasma from current and former smokers suppressed CFTR chloride transport [33]. Together, these studies indicate that smoke exposure can distribute through the circulatory system, leading to systemic CFTR impairment.

Interestingly, unlike in the airways, extrapulmonary CFTR dysfunction in former smokers may not recover despite smoking cessation $[32,33]$. The reason for this discrepancy is not known and deserves additional study. A possible explanation may lie in the fact that sweat chloride is a relatively sensitive test and may be able to detect subtle changes in CFTR function that may otherwise go unobserved in the respiratory epithelium [62]. Other proposed mechanisms for the sustained effect include tissue retention and sustained release of cigarette smoke containing toxins such as acrolein [63] as well as the formation of acrolein-modified proteins which have been shown to persist despite smoking cessation [64].

Several mechanisms have been identified by which smoke exposure impairs wild-type CFTR function and expression. Smoke exposure induces a CFTR gating defect $[65,66]$, decreasing open-channel probability by $\sim 75 \%$ [65] with a concomitant $50 \%$ reduction in chloride conductance [65]. This defect occurs rapidly after exposure and partially recovers post-exposure $[60,66]$. Additionally, cigarette smoke decreases channel expression [27] by suppressing CFTR-encoding mRNA [28, 31], decreasing new CFTR protein synthesis [60], and sequestering CFTR to perinuclear, aggresome-like bodies, which, unlike lysosome bodies, are not immediately degraded [60]. Suppression of CFTR protein and mRNA levels are observed within minutes [60] and hours [28], respectively, after smoke exposure and with as few as three puff equivalents of tobacco smoke $[60,66]$. The degree to which each of these mechanisms contributes to the overall channel dysfunction is not fully understood but may explain both immediate impairment and delayed recovery with smoke exposure.

Noting that cigarette smoke consists of multiple toxins, there is ongoing investigation into the contribution of its individual constituents to CFTR dysfunction. A key body of evidence links smoke-induced CFTR impairment to the component acrolein, a highly reactive molecule that is found in elevated levels in smokers [33, 65, 67]. Acrolein has been shown to directly modify CFTR protein [33, 65, 67] and rapidly induce a CFTR gating defect [33], reducing forskolin-stimulated (CFTR dependent) short-circuit current in a dose-dependent fashion [33]. With implications on therapeutic approaches, the reducing agent $\mathrm{N}$-acetyl-cysteine (NAC) has been observed to be protective against the deleterious effects of acrolein on CFTR [33]. NAC has been evaluated as a treatment for COPD with mixed results but at doses that do not achieve serum or tissue levels adequate to abrogate acrolein-induced effects $[33,68]$.

The heavy metal cadmium, another component of cigarette smoke that reaches detectable levels in the serum of smokers $[69,70]$, has also been linked to smoke-induced CFTR dysfunction through inhibitory effects on CFTR mRNA and protein expression, resulting in decreased chloride transport [58]. The fat-soluble anti-oxidant $\alpha$-tocopherol, although not NAC, was protective against cadmium-mediated CFTR impairment, plausibly by stabilising CFTR protein in the plasma membrane [58].

Epidemiological studies evaluating the rate of CFTR mutations among patients with COPD suggest that genetic contributions to acquired CFTR dysfunction are less substantial than environmental causes. Early studies indicated that the frequency of CFTR mutations may be elevated in this population [71, 72], although sample sizes were small and, in one study, the population screened consisted of chronic bronchitis patients with simultaneous diffuse bronchiectasis [72]. This association was not evident in more recent, larger studies [73-76].

\section{CFTR dysfunction and asthma}

While asthma and cystic fibrosis are generally considered unrelated disorders, they share several similar features including wheezing, cough and recurrent airway obstruction [77, 78]. Asthma is typified by an eosinophilic and T-helper cell (Th)2-type inflammatory profile, although a neutrophilic (Th1) endotype is being increasingly recognised [78, 79] and correlates with asthma severity [80-82]. Case reports of neutrophilic asthma show that this population demonstrates additional clinical manifestations seen in people with cystic fibrosis, including bronchiectasis, recurrent airway infections and infections with 
common cystic fibrosis pathogens such as Pseudomonas spp. and Staphylococcus aureus [78, 83]. In addition, severe asthma and fatal exacerbations are associated with prominent changes in mucus expression and composition resembling cystic fibrosis $[2,84]$.

These similarities have inspired investigations into the relationship between CFTR, cystic fibrosis and neutrophilic asthma. In clinical evaluations of patients with neutrophilic asthma, sweat chloride was elevated to a level consistent with a mild-to-intermediate CFTR impairment $[78,83]$. In one study, NPD was consistently abnormal, some parameters in the cysti fibrosis range, leading the authors to suggest that some forms of asthma represent a cystic fibrosis-related disease [83]. Epidemiological studies assessing rates of asthma among cystic fibrosis carriers have yielded mixed results, although variation of findings may be associated with inadequate endotyping of the asthmatic inflammation. Some studies found a higher prevalence of asthma among F508del heterozygotes [85] and higher rates of carrier status among asthmatics [86], while others did not [87, 88]. One study suggests carrier status to be protective against developing asthma [89]. While these studies primarily focused on association with F508del, complete CFTR analysis in asthmatics confirmed a higher rate of variants and polymorphisms, including the gain-of-function variant M470V [75, 90]. In this population, the M470V variant was found in $90 \%$ of asthmatics that had a CFTR mutations [90], underscoring a putative relationship between asthma and CFTR mutations and CFTR variants. Complete gene analysis may more accurately delineate the relationship. Together, findings to date indicate a potential role for acquired CFTR dysfunction in neutrophilic endotype asthma, although further studies are needed to better delineate this relationship. Similarly, while CFTR dysfunction offers a plausible mechanism to explain the mucus changes and plugging found in severe asthma [2, 84], investigation is also needed to confirm this mechanism.

\section{CFTR dysfunction in allergic bronchopulmonary aspergillosis}

Allergic bronchopulmonary aspergillosis (ABPA) is a hypersensitivity reaction to Aspergillus fumigatus colonisation of the airways causing symptoms of uncontrolled asthma as well as central bronchiectasis with mucus plugging. Given the similarities to cystic fibrosis, several investigators have looked for evidence of CFTR mutations in this population. While studies were limited by small patient size, patients with ABPA were more likely to have CFTR mutations than the general population [91]. A more recent investigation that screened for a more diverse set of CFTR mutations found almost two-thirds of the patients were carriers of CFTR mutations and over one-third of the patients had mild elevations in sweat chloride levels [92]. While there appears to be an association of CFTR mutations and ABPA, further investigation is needed to understand if CFTR is dysfunctional and the degree to which it contributes to the development or manifestations of ABPA.

\section{CFTR dysfunction in bronchiectasis}

Bronchiectasis is a disorder characterised by pathological inflammation and dilation of the airways, leading to impaired mucin clearance, chronic sputum production and recurrent infections. Bronchiectasis can result from a number of insults, although in about half the cases, an aetiology cannot be determined [93]. Idiopathic bronchiectasis (IB) tends to have a more indolent course than cystic fibrosis [94].

Clinically, there is striking overlap of features between the two disorders. Both have a prominent, productive cough with recurrent exacerbations and are similarly driven by chronic neutrophilic inflammation with increased levels of neutrophil-recruiting cytokines [95]. Additionally, the reduced mucociliary clearance seen in idiopathic bronchiectasis results in increased risk of colonisation with virulent pathogens common to cystic fibrosis such as Pseudomonas spp., Staphylococcus aureus, non-tuberculous Mycobacterium spp., and moulds such as Aspergillus spp. [93, 94, 96]. These clinical similarities would support similar pathologic mechanisms, which may include CFTR dysfunction.

There is mixed evidence for the role of CFTR mutations in idiopathic bronchiectasis [97-101]. Differences in genetic testing and small sample size likely accounts for difficulty detecting an association between CFTR mutations and idiopathic bronchiectasis [97, 98]. Other studies have found an association [101], and with more extensive genetic testing, idiopathic bronchiectasis was associated with several missense mutations, polymorphisms and variants of CFTR $[75,99,100]$ that, when present, influence CFTR function and expression [98, 102]. These studies found that $26-51 \%$ of patients with idiopathic bronchiectasis harboured CFTR mutations, which is higher than expected compared with carrier frequency in the general population [75, 98-101] suggesting an association.

CFTR conductance is impaired in idiopathic bronchiectasis in proportion with the number of mutant alleles. In support, multiple studies have documented that this population has elevated sweat chloride levels $[97,98,101]$, and that those with mutations on both alleles have higher sweat chloride than those with just one mutant allele $[98,102]$. NPD measurements in patients with idiopathic bronchiectasis who carried zero, one or two CFTR mutations also demonstrated CFTR impairment correlating with the 
number of mutant alleles, with a degree of dysfunction ranging between that seen in healthy controls and patients with cystic fibrosis [102]. These findings are consistent with others reporting that there is a continuum of CFTR dysfunction ranging from cystic fibrosis-related diseases to severe cystic fibrosis [33, 61]. Taken together, these studies indicate possible disproportionate CFTR dysfunction in idiopathic bronchiectasis compared with asymptomatic carriers of CFTR mutations. It remains unclear whether non-CFTR mutations that disrupt CFTR processing and/or function or that the process is secondary to the inflammatory environment caused by CFTR dysfunction is not yet clear.

One challenge in assessing the association between the number of mutant alleles and CFTR dysfunction in idiopathic bronchiectasis is that disease-modifying variants influence expression in ways that are difficult to predict. For instance, one variant found in idiopathic bronchiectasis, 5T, variably decreases CFTR synthesis $[99,102]$ while another, M470V, increases conductance almost two-fold over the native allele and has a causal role in cystic fibrosis that remains in question [98, 100, 103]. Given these complicated interactions, it is understandably difficult to accurately predict idiopathic bronchiectasis phenotype based on genotype. Consistent with other cystic fibrosis-related disorders, genetic tests were shown to have a low sensitivity for diagnosis in the absence of sequencing [61].

The complicated genotype-phenotype relationship may account for the heterogeneity in CFTR dysfunction observed in idiopathic bronchiectasis. For example, subjects with idiopathic bronchiectasis and one CFTR mutation had measurable CFTR dysfunction, while obligate heterozygotes did not, despite the same number of mutant alleles [102]. Even among those with the same mutation, there was a variable amount of CFTR dysfunction, including in carriers of severe CFTR mutations like F508del [100, 102]. These studies suggest that while CFTR mutation may contribute to the development of idiopathic bronchiectasis, the variability of CFTR dysfunction may be a function of additional unidentified factors that induce secondary acquired reductions in CFTR activity [102]. Other shared mechanisms of disease pathogenesis between cystic fibrosis and idiopathic bronchiectasis may, therefore, be responsible for CFTR dysfunction as a "second hit" in carriers.

In conclusion, while there is a clear association between CFTR dysfunction and idiopathic bronchiectasis, further studies are needed to fully understand its pathogenic contribution. It is not clear if the channelopathy primarily drives the formation of bronchiectasis, or if the channelopathy makes an individual more susceptible only after a second insult such as smoke or neutrophilic inflammation. More research is needed to better understand the genetic factors that cause the variability in channel dysfunction seen in current studies. As our understanding of CFTR dysfunction and shared pathophysiology between cystic fibrosis and idiopathic bronchiectasis grows, we may better understand if CFTR modulators will benefit this patient population.

\section{CFTR modulators in primary ciliary dyskinesia}

In addition to idiopathic forms of bronchiectasis, primary ciliary dyskinesia (PCD) demonstrates pulmonary (e.g. bronchiectasis and chronic infections) and sinus (e.g. chronic rhinosinusitis) phenotypic similarities to cystic fibrosis. While there are no studies directly demonstrating CFTR dysfunction in PCD patients, there is evidence that CFTR chloride conduction can be regulated by local purinergic signalling [104] by motile cilia in response to changes in mucus viscosity [36]. As mucus becomes more dehydrated and viscous, mechanical strain sense by the cilia stimulates ATP release [36] which, through local purinergic receptors, stimulates anion transporters including CFTR [104] leading to mucus hydration and restoring the airway surface liquid (ASL) depth [36]. However, PCD cell cultures with non-motile cilia had no response to increased mucus viscosity demonstrating motile cilia are integral to the process [36]. These findings suggest that mucus viscosity can be locally regulated through feedback involving CFTR but is vitally dependent on motile cilia. By inference, local CFTR function may be decreased by the lack of purinergic drive from motile cilia in patients with PCD. Further studies are needed to elucidate what role, if any, CFTR dysfunction contributes to PCD.

\section{CFTR modulators in COPD and chronic bronchitis}

Evidence of CFTR dysfunction in COPD with chronic bronchitis raises the possibility of novel treatment strategies for this population. Inhaled therapies are the mainstay of management for COPD and, while symptoms, hospitalisation frequency, and exacerbation rates may improve with inhaled corticosteroids and bronchodilators, reductions in mortality have yet not been observed [105-107]. Only smoking cessation and supplemental oxygen for severe hypoxaemia have been shown to impact mortality [108-111]. Similarly, mucolytics have demonstrated only marginal benefit $[68,112,113]$.

The phosphodiesterase inhibitor, roflumilast, was initially targeted for its proposed anti-inflammatory effects in COPD and was shown to reduce exacerbations in a subset of patients with chronic bronchitis who had persistent exacerbations $[114,115]$. Subsequent studies found that roflumilast stimulates CFTR-dependent chloride transport through an increase in intracellular cAMP-mediated CFTR phosphorylation and can abrogate smoke-induced CFTR dysfunction $[114,116,117]$. The effects of roflumilast on CFTR, rather than 
its anti-inflammatory properties, may explain why benefit was limited to the patient population with chronic bronchitis $[118,119]$ and point toward the potential of CFTR modulation as a therapeutic modality in COPD.

Indeed, although few studies have evaluated the efficacy of established CFTR modulators in smoking-induced CFTR dysfunction, findings to date support this strategy [31, 65, 114]. Ivacaftor has previously been shown to stimulate wild-type CFTR, indicative of a role for potentiator approaches in disorders of acquired CFTR dysfunction [31]. Ivacaftor was also shown to partially reverse smoke-induced changes in human bronchial epithelial cell cultures $[31,65,114]$ by augmenting open-channel probability [65], improving chloride conductance [31, 65], restoring ASL [31, 65], and improving MCT [31]. Similar improvements were found in cystic fibrosis models treated with ivacaftor [42], consistent with a shared pathology and further supporting the potential for treatment of COPD with CFTR potentiators.

Clinical trials testing CFTR potentiators in COPD are in the early stages. A recently completed pilot study evaluating ivacaftor in COPD patients with chronic bronchitis showed a trend towards improved symptoms and sweat chloride levels, as well as a $20 \%$ improvement in CFTR activity, with no safety concerns noted [120]. A follow-up study is underway examining ivacaftor on longer-term outcomes [121]. Complementing these data, a phase 2 trial assessing the effects of the investigational CFTR potentiator, QBW251 (Novartis Pharmaceuticals), has been conducted [56]. After 28 days of treatment, patients demonstrated improvements in sweat chloride, spirometry, inflammatory markers and sputum colonisation, although no difference was found in the primary outcome of lung clearance index [56]. The effect of CFTR modulators in these two clinical trials is summarised in figure 1 compared with responses of CFTR modulators in various CFTR mutation types. Ivacaftor has also been tested in PCD in combination with a novel ENaC inhibitor (VX-371; Vertex Pharmaceuticals) [122]. At present, this trial is fully enrolled, with the results of the phase 2 trial expected in 2020 [122].

\section{Conclusions}

This review examines the role of CFTR protein dysfunction in disorders of the respiratory tract. Significant progress in the development of highly effective CFTR modulators gives promise for improved outcomes in patients with cystic fibrosis. In addition, a heightened understanding of other chronic diseases of the airways has linked acquired CFTR dysfunction to the pathophysiology of these disease states (figure 2). An overview of clinical trials CFTR modulators in non-cystic fibrosis lung disease is presented in table 1. With further development, CFTR modulators may prove to be a therapy for some lung diseases associated with acquired CFTR dysfunction or impaired mucociliary clearance.
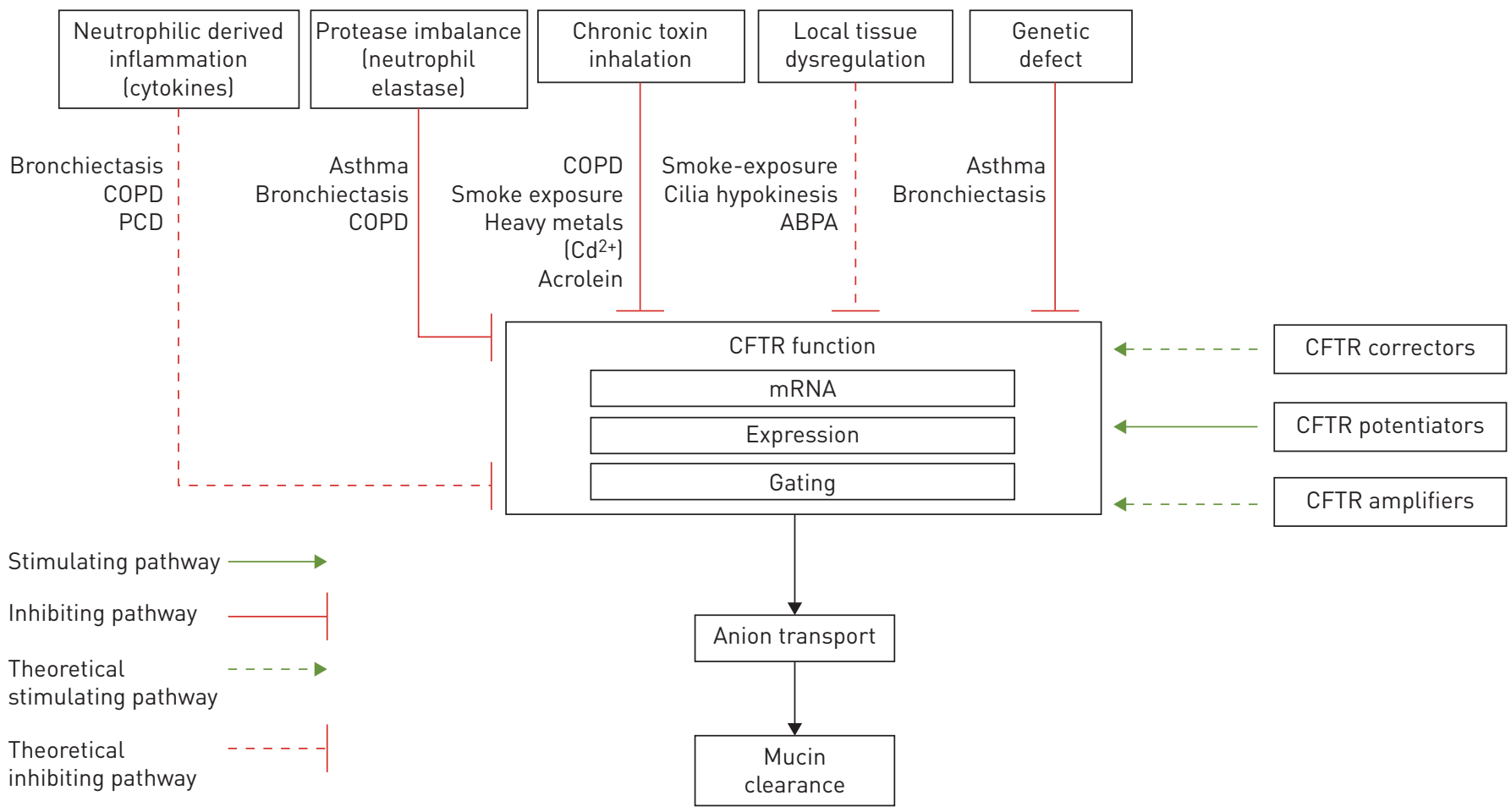

FIGURE 2 Schematic representation of potential and known mechanisms influencing cystic fibrosis transmembrane conductance regulator (CFTR) function. PCD: primary ciliary dyskinesia; ABPA: allergic bronchopulmonary aspergillosis. 
TABLE 1 Overview of clinical trials assessing cystic fibrosis transmembrane conductance regulator (CFTR) modulators in non-cystic fibrosis lung disease

\begin{tabular}{|c|c|c|c|c|c|}
\hline Study name/NCT & Author [ref.] & Population & Drug & Results & Sponsor \\
\hline $\begin{array}{l}\text { Cystic fibrosis transmembrane } \\
\text { conductance regulator activation by } \\
\text { roflumilast contributes to therapeutic } \\
\text { benefit in chronic bronchitis }\end{array}$ & LAMBERT [114] & $\begin{array}{l}\text { In vitro: } \mathrm{HBE} \\
\text { smoke exposed } \\
\text { cells }\end{array}$ & $\begin{array}{l}\text { Roflumilast } \\
\text { and Ivacaftor }\end{array}$ & $\begin{array}{l}\text { Roflumilast restores CFTR } \\
\text { activity, ASL recovery and height } \\
\text { post smoke exposure in vitro. } \\
\text { Effect was additive with ivacaftor. }\end{array}$ & \\
\hline $\begin{array}{l}\text { Roflumilast combined with adenosine } \\
\text { increases mucosal hydration in } \\
\text { human airway epithelial cultures } \\
\text { after cigarette smoke exposure }\end{array}$ & TYRRELL [116] & $\begin{array}{l}\text { In vitro: } \mathrm{HBE} \\
\text { smoke exposed } \\
\text { cells }\end{array}$ & Roflumilast & $\begin{array}{l}\text { Roflumilast improved cellular } \\
\text { cAMP and ASL height recovery } \\
\text { post smoke exposure. }\end{array}$ & \\
\hline $\begin{array}{l}\text { A pharmacologic approach to acquired } \\
\text { cystic fibrosis transmembrane } \\
\text { conductance regulator dysfunction in } \\
\text { smoking related lung disease. }\end{array}$ & SLoAne [31] & $\begin{array}{l}\text { In vitro: HBE } \\
\text { In vivo: Smokers } \\
\text { with and without } \\
\text { COPD }\end{array}$ & Ivacaftor & $\begin{array}{l}\text { Ivacaftor activated CFTR } \\
\text { transport, reduced smoked } \\
\text { induced impairment in vitro. }\end{array}$ & \\
\hline $\begin{array}{l}\text { Pilot evaluation of ivacaftor for chronic } \\
\text { bronchitis }\end{array}$ & $\begin{array}{l}\text { SOLOMON } \\
\text { [120] }\end{array}$ & $\begin{array}{l}\text { Chronic } \\
\text { bronchitis: pilot } \\
\text { study }\end{array}$ & Ivacaftor & $\begin{array}{l}\text { Trend towards improved } \\
\text { symptoms and sweat chloride. }\end{array}$ & \\
\hline Topic Trial NCT02135432 & Rowe [121] & COPD & Ivacaftor & Results pending & \\
\hline $\begin{array}{l}\text { A randomized, placebo controlled } \\
\text { 4-week study in COPD of QBW251, a } \\
\text { potentiator of the CFTR protein }\end{array}$ & Rowe [56] & COPD & QWB251 & $\begin{array}{l}\text { No improvement in lung } \\
\text { clearance index. Improved sweat } \\
\text { chloride, spirometry, } \\
\text { inflammation, and sputum } \\
\text { colonisation (figure 1). }\end{array}$ & Novartis \\
\hline
\end{tabular}

Acknowledgements: S.D. Patel, T.R. Bono, S.M. Rowe and G.M. Solomon each contributed equally to authorship of the manuscript.

Conflict of interest: S.D. Patel has nothing to disclose. T.R. Bono has nothing to disclose. S.M. Rowe reports grants from Bayer, Forest Research Institute, AstraZeneca, N30/Nivalis, Novartis, Galapagos/AbbVie, Proteostasis, Eloxx and PTC Therapeutics, grants and personal fees from Celtaxsys, grants, personal fees and non-financial support from Vertex Pharmaceuticals Incorporated, and personal fees from Bayer and Novartis, outside the submitted work. G.M. Solomon reports grants from NIH and the CF Foundation, during the conduct of the study; grants and personal fees from Vertex Pharamceuticals, Electromed, Inc. and Insmed Inc., and grants from Saavara, Inc. and Parion Sciences, outside the submitted work.

Support statement: Funding support has been received from the National Institutes of Health National Institute of Diabetes and Digestive and Kidney Diseases (P30-2P30DK072482 to S.M. Rowe and G.M. Solomon), National Institutes of Health (1K08-HL138153 to G.M. Solomon and R35HL135816 to S.M. Rowe) and Cystic Fibrosis Foundation (Solomon18YO) to G.M. Solomon. Funding information for this article has been deposited with the Crossref Funder Registry.

\section{References}

1 O'Sullivan BP, Freedman SD. Cystic fibrosis. Lancet 2009; 373: 1891-1904.

2 Fahy JV, Dickey BF. Airway mucus function and dysfunction. N Engl J Med 2010; 363: 2233-2247.

3 Knowles MR, Durie PR. What is cystic fibrosis? N Engl J Med 2002; 347: 439-442.

4 Redente EF, Jakubzick CV, Martin TR, et al. Innate Immunity. In: Broaddus VC, Mason RJ, Ernst JD, et al. eds.

Murray and Nadel's Textbook of Respiratory Medicine. Philadelphia, Elsevier, 2016; pp. 184-205. 
Birket SE, Davis JM, Fernandez CM, et al. Development of an airway mucus defect in the cystic fibrosis rat. JCI Insight 2018; 3: e97199.

6 Borowitz D. CFTR, bicarbonate, and the pathophysiology of cystic fibrosis. Pediatr Pulmonol 2015; 50: Suppl. 40, S24-S30.

7 Gorrieri G, Scudieri P, Caci E, et al. Goblet cell hyperplasia requires high bicarbonate transport to support mucin release. Sci Rep 2016; 6: 36016 .

Pezzulo AA, Tang XX, Hoegger MJ, et al. Reduced airway surface $\mathrm{pH}$ impairs bacterial killing in the porcine cystic fibrosis lung. Nature 2012; 487: 109-113.

9 Lazrak A, Jurkuvenaite A, Chen L, et al. Enhancement of alveolar epithelial sodium channel activity with decreased cystic fibrosis transmembrane conductance regulator expression in mouse lung. Am J Physiol Lung Cell Mol Physiol 2011; 301: L557-L567.

10 Zhou-Suckow Z, Duerr J, Hagner M, et al. Airway mucus, inflammation and remodeling: emerging links in the pathogenesis of chronic lung diseases. Cell Tissue Res 2017; 367: 537-550.

11 Mall MA, Galietta LJ. Targeting ion channels in cystic fibrosis. J Cyst Fibros 2015; 14: 561-570.

12 Zhou Z, Duerr J, Johannesson B, et al. The ENaC-overexpressing mouse as a model of cystic fibrosis lung disease. J Cyst Fibros 2011; 10: Suppl. 2, S172-S182.

13 Peabody JE, Shei RJ, Bermingham BM, et al. Seeing cilia: imaging modalities for ciliary motion and clinical connections. Am J Physiol Lung Cell Mol Physiol 2018; 314: L909-L921.

14 Liu L, Shastry S, Byan-Parker S, et al. An autoregulatory mechanism governing mucociliary transport is sensitive to mucus load. Am J Respir Cell Mol Biol 2014; 51: 485-493.

15 Matsui H, Wagner VE, Hill DB, et al. A physical linkage between cystic fibrosis airway surface dehydration and Pseudomonas aeruginosa biofilms. Proc Natl Acad Sci USA 2006; 103: 18131-18136.

16 Mall M, Grubb BR, Harkema JR, et al. Increased airway epithelial Na+ absorption produces cystic fibrosis-like lung disease in mice. Nat Med 2004; 10: 487-493.

17 Clinical and Functional TRanslation of CFTR (CFTR2). http://cftr2.org Date last accessed: 1 March 2020; date last updated: 1 March 2020.

18 Rowe SM, Miller S, Sorscher EJ. Cystic fibrosis. N Engl J Med 2005; 352: 1992-2001.

19 Rafeeq MM, Murad HAS. Cystic fibrosis: current therapeutic targets and future approaches. J Transl Med 2017; 15: 84.

20 Davis PB. Cystic fibrosis since 1938. Am J Respir Crit Care Med 2006; 173: 475-482.

21 Solomon GM, Marshall SG, Ramsey BW, et al. Breakthrough therapies: Cystic fibrosis (CF) potentiators and correctors. Pediatr Pulmonol 2015; 50: Suppl. 40, S3-S13.

22 Cutting GR. Cystic fibrosis genetics: from molecular understanding to clinical application. Nat Rev Genet 2015; 16: 45-56.

23 Serohijos AW, Hegedus T, Aleksandrov AA, et al. Phenylalanine-508 mediates a cytoplasmic-membrane domain contact in the CFTR 3D structure crucial to assembly and channel function. Proc Natl Acad Sci USA 2008; 105: 3256-3261.

24 Jurkuvenaite A, Varga K, Nowotarski K, et al. Mutations in the amino terminus of the cystic fibrosis transmembrane conductance regulator enhance endocytosis. J Bio Chem 2006; 281: 3329-3334.

25 Castellani C, Cuppens H, Macek M Jr, et al. Consensus on the use and interpretation of cystic fibrosis mutation analysis in clinical practice. J Cyst Fibros 2008; 7: 179-196.

26 Welsh MJ. Cigarette smoke inhibition of ion transport in canine tracheal epithelium. J Clin Invest 1983; 71: 1614-1623.

27 Dransfield MT, Wilhelm AM, Flanagan B, et al. Acquired cystic fibrosis transmembrane conductance regulator dysfunction in the lower airways in COPD. Chest 2013; 144: 498-506.

28 Cantin AM, Hanrahan JW, Bilodeau G, et al. Cystic fibrosis transmembrane conductance regulator function is suppressed in cigarette smokers. Am J Respir Crit Care Med 2006; 173: 1139-1144.

29 Kreindler JL, Jackson AD, Kemp PA, et al. Inhibition of chloride secretion in human bronchial epithelial cells by cigarette smoke extract. Am J Physiol Lung Cell Mol Physiol 2005; 288: L894-L902.

30 Maestrelli P, Saetta M, Mapp CE, et al. Remodeling in response to infection and injury. Airway inflammation and hypersecretion of mucus in smoking subjects with chronic obstructive pulmonary disease. Am J Respir Crit Care Med 2001; 164: Pt 2, S76-S80.

31 Sloane PA, Shastry S, Wilhelm A, et al. A pharmacologic approach to acquired cystic fibrosis transmembrane conductance regulator dysfunction in smoking related lung disease. PLoS One 2012; 7: e39809.

32 Courville CA, Tidwell S, Liu B, et al. Acquired defects in CFTR-dependent beta-adrenergic sweat secretion in chronic obstructive pulmonary disease. Respir Res 2014; 15: 25.

33 Raju SV, Jackson PL, Courville CA, et al. Cigarette smoke induces systemic defects in cystic fibrosis transmembrane conductance regulator function. Am J Respir Crit Care Med 2013; 188: 1321-1330.

34 Saint-Criq V, Gray MA. Role of CFTR in epithelial physiology. Cell Mol Life Sci 2017; 74: 93-115.

35 Le Gars M, Descamps D, Roussel D, et al. Neutrophil elastase degrades cystic fibrosis transmembrane conductance regulator via calpains and disables channel function in vitro and in vivo. Am J Respir Crit Care Med 2013; 187: 170-179.

36 Button B, Okada SF, Frederick CB, et al. Mechanosensitive ATP release maintains proper mucus hydration of airways. Sci Signal 2013; 6: ra46.

37 Button B, Cai LH, Ehre C, et al. A periciliary brush promotes the lung health by separating the mucus layer from airway epithelia. Science 2012; 337: 937-941.

38 Brown SD, White R, Tobin P. Keep them breathing: Cystic fibrosis pathophysiology, diagnosis, and treatment. JAAPA 2017; 30: 23-27.

39 Flume PA, Mogayzel PJ Jr, Robinson KA, et al. Cystic fibrosis pulmonary guidelines: treatment of pulmonary exacerbations. Am J Respir Crit Care Med 2009; 180: 802-808.

40 Blease K, Burke-Gaffney A, Hellewell PG. Modulation of cell adhesion molecule expression and function on human lung microvascular endothelial cells by inhibition of phosphodiesterases 3 and 4. Br J Pharmacol 1998; 124: $229-237$.

41 Donaldson SH, Corcoran TE, Laube BL, et al. Mucociliary clearance as an outcome measure for cystic fibrosis clinical research. Proc Am Thorac Soc 2007; 4: 399-405. 
42 Van Goor F, Hadida S, Grootenhuis PD, et al. Rescue of CF airway epithelial cell function in vitro by a CFTR potentiator, VX-770. Proc Natl Acad Sci USA 2009; 106: 18825-18830.

43 Ramsey BW, Davies J, McElvaney NG, et al. A CFTR potentiator in patients with cystic fibrosis and the G551D mutation. N Engl J Med 2011; 365: 1663-1672.

44 Yu H, Burton B, Huang CJ, et al. Ivacaftor potentiation of multiple CFTR channels with gating mutations. J Cyst Fibros 2012; 11: 237-245.

45 De Boeck K, Munck A, Walker S, et al. Efficacy and safety of ivacaftor in patients with cystic fibrosis and a non-G551D gating mutation. J Cyst Fibros 2014; 13: 674-680.

46 FDA expands approved use of Kalydeco to treat additional mutations of cystic fibrosis [press release]. US Food and Drug Administration, 2017. https://www.fda.gov/news-events/press-announcements/fda-expands-approveduse-kalydeco-treat-additional-mutations-cystic-fibrosis Date last accessed: 15 February 2020; date last updated: 15 February 2020.

47 Durmowicz AG, Lim R, Rogers H, et al. The U.S. Food and Drug Administration's experience with ivacaftor in cystic fibrosis. establishing efficacy using in vitro data in lieu of a clinical trial. Ann Am Thorac Soc 2018; 15: 1-2.

48 Van Goor F, Straley KS, Cao D, et al. Rescue of DeltaF508-CFTR trafficking and gating in human cystic fibrosis airway primary cultures by small molecules. Am J Physiol Lung Cell Mol Physiol 2006; 290: L1117-L1130.

49 Boyle MP, Bell SC, Konstan MW, et al. A CFTR corrector (lumacaftor) and a CFTR potentiator (ivacaftor) for treatment of patients with cystic fibrosis who have a phe508del CFTR mutation: a phase 2 randomised controlled trial. Lancet Respir Med 2014; 2: 527-538.

50 Wainwright CE, Elborn JS, Ramsey BW. Lumacaftor-ivacaftor in patients with cystic fibrosis homozygous for Phe508del CFTR. N Engl J Med 2015; 373: 1783-1784.

51 Taylor-Cousar JL, Munck A, McKone EF, et al. Tezacaftor-ivacaftor in patients with cystic fibrosis homozygous for Phe508del. N Engl J Med 2017; 377: 2013-2023.

52 Rowe SM, Daines C, Ringshausen FC, et al. Tezacaftor-ivacaftor in residual-function heterozygotes with cystic fibrosis. N Engl J Med 2017; 377: 2024-2035.

53 Heijerman HGM, McKone EF, Downey DG, et al. Efficacy and safety of the elexacaftor plus tezacaftor plus ivacaftor combination regimen in people with cystic fibrosis homozygous for the F508del mutation: a double-blind, randomised, phase 3 trial. Lancet 2019; 394: 1940-1948.

54 Middleton PG, Mall MA, Drevinek P, et al. Elexacaftor-tezacaftor-ivacaftor for cystic fibrosis with a single Phe508del allele. N Engl J Med 2019; 381: 1809-1819.

55 Rowe SM, Accurso F, Clancy JP. Detection of cystic fibrosis transmembrane conductance regulator activity in early-phase clinical trials. Proc Am Thorac Soc 2007; 4: 387-398.

56 Rowe SM, Haque N, Gleason S, et al. A randomized, placebo controlled 4-week study in COPD of QBW251, a potentiator of the cystic fibrosis transmembrane conductance regulator (CFTR) protein. Eur Repir J 2018; 52: Suppl. 62, PA617.

57 Raju SV, Solomon GM, Dransfield MT, et al. Acquired cystic fibrosis transmembrane conductance regulator dysfunction in chronic bronchitis and other diseases of mucus clearance. Clin Chest Med 2016; 37: 147-158.

58 Rennolds J, Butler S, Maloney $\mathrm{K}$, et al. Cadmium regulates the expression of the CFTR chloride channel in human airway epithelial cells. Toxicol Sci 2010; 116: 349-358.

59 Brand JD, Lazrak A, Trombley JE, et al. Influenza-mediated reduction of lung epithelial ion channel activity leads to dysregulated pulmonary fluid homeostasis. JCI Insight 2018; 3: e123467.

60 Clunes LA, Davies CM, Coakley RD, et al. Cigarette smoke exposure induces CFTR internalization and insolubility, leading to airway surface liquid dehydration. Faseb J 2012; 26: 533-545.

61 Wilschanski M, Dupuis A, Ellis L, et al. Mutations in the cystic fibrosis transmembrane regulator gene and in vivo transepithelial potentials. Am J Respir Crit Care Med 2006; 174: 787-794.

62 Solomon GM, Raju SV, Dransfield MT, et al. Therapeutic approaches to acquired cystic fibrosis transmembrane conductance regulator dysfunction in chronic bronchitis. Ann Am Thorac Soc 2016; 13: Suppl. 2, S169-S176.

63 Uchida K, Kanematsu M, Sakai K, et al. Protein-bound acrolein: potential markers for oxidative stress. Proc Natl Acad Sci USA 1998; 95: 4882-4887.

64 Kitaguchi Y, Taraseviciene-Stewart L, Hanaoka M, et al. Acrolein induces endoplasmic reticulum stress and causes airspace enlargement. PLoS One 2012; 7: e38038.

65 Raju SV, Lin VY, Liu L, et al. The cystic fibrosis transmembrane conductance regulator potentiator ivacaftor augments mucociliary clearance abrogating cystic fibrosis transmembrane conductance regulator inhibition by cigarette smoke. Am J Respir Cell Mol Biol 2017; 56: 99-108.

66 Moran AR, Norimatsu Y, Dawson DC, et al. Aqueous cigarette smoke extract induces a voltage-dependent inhibition of CFTR expressed in Xenopus oocytes. Am J Physiol Lung Cell Mol Physiol 2014; 306: L284-L291.

67 Lin VY, Fain MD, Jackson PL, et al. Vaporized E-cigarette liquids induce ion transport dysfunction in airway epithelia. Am J Respir Cell Mol Biol 2019; 61: 162-173.

68 Decramer M, Rutten-van Molken M, Dekhuijzen PN, et al. Effects of N-acetylcysteine on outcomes in chronic obstructive pulmonary disease (Bronchitis Randomized on NAC Cost-Utility Study, BRONCUS): a randomised placebo-controlled trial. Lancet 2005; 365: 1552-1560.

69 Paakko P, Kokkonen P, Anttila S, et al. Cadmium and chromium as markers of smoking in human lung tissue. Environ Res 1989; 49: 197-207.

70 Rokadia HK, Agarwal S. Serum heavy metals and obstructive lung disease: results from the National Health and Nutrition Examination Survey. Chest 2013; 143: 388-397.

71 Dumur V, Lafitte JJ, Gervais R, et al. Abnormal distribution of cystic fibrosis delta F508 allele in adults with chronic bronchial hypersecretion. Lancet 1990; 335: 1340.

72 Poller W, Faber JP, Scholz S, et al. Sequence analysis of the cystic fibrosis gene in patients with disseminated bronchiectatic lung disease. Application in the identification of a cystic fibrosis patient with atypical clinical course. Klin Wochenschr 1991; 69: 657-663.

73 Gervais R, Lafitte JJ, Dumur V, et al. Sweat chloride and delta F508 mutation in chronic bronchitis or bronchiectasis. Lancet 1993; 342: 997.

74 Raju SV, Tate JH, Peacock SK, et al. Impact of heterozygote CFTR mutations in COPD patients with chronic bronchitis. Respir Res 2014; 15: 18. 
Tzetis M, Efthymiadou A, Strofalis S, et al. CFTR gene mutations - including three novel nucleotide substitutions and haplotype background in patients with asthma, disseminated bronchiectasis and chronic obstructive pulmonary disease. Hum Genet 2001; 108: 216-221.

Artlich A, Boysen A, Bunge S, et al. Common CFTR mutations are not likely to predispose to chronic bronchitis in northern Germany. Hum Genet 1995; 95: 226-228.

Nakagome K, Matsushita S, Nagata M. Neutrophilic inflammation in severe asthma. Int Arch Allergy Immunol 2012; 158: Suppl. 1, 96-102.

Goodwin J, Spitale N, Yaghi A, et al. Cystic fibrosis transmembrane conductance regulator gene abnormalities in patients with asthma and recurrent neutrophilic bronchitis. Can Respir J 2012; 19: 46-48.

Peebles RS Jr, Aronica MA. Proinflammatory pathways in the pathogenesis of asthma. Clin Chest Med 2019; 40: 29-50.

Marguet C, Jouen-Boedes F, Dean TP, et al. Bronchoalveolar cell profiles in children with asthma, infantile wheeze, chronic cough, or cystic fibrosis. Am I Respir Crit Care Med 1999; 159(5 Pt 1): 1533-1540.

Bullens DM, Truyen E, Coteur L, et al. IL-17 mRNA in sputum of asthmatic patients: linking T cell driven inflammation and granulocytic influx? Respir Res 2006; 7: 135.

Doe C, Bafadhel M, Siddiqui S, et al. Expression of the T helper 17-associated cytokines IL-17A and IL-17F in asthma and COPD. Chest 2010; 138: 1140-1147.

Schulz A, Tummler B. Non-allergic asthma as a CFTR-related disorder. J Cyst Fibros 2016; 15: 641-644.

Sheehan JK, Richardson PS, Fung DC, et al. Analysis of respiratory mucus glycoproteins in asthma: a detailed study from a patient who died in status asthmaticus. Am J Respir Cell Mol Biol 1995; 13: 748-756.

Lowenfels AB, Maisonneuve P, Palys B, et al. DeltaF508 heterozygosity and asthma. Lancet 1998; 352: 985; author reply 986-987.

Dahl M, Tybjaerg-Hansen A, Lange P, et al. DeltaF508 heterozygosity in cystic fibrosis and susceptibility to asthma. Lancet 1998; 351: 1911-1913.

Kauffmann F, Chomel JC, Kitzis A, et al. DeltaF508 heterozygosity and asthma. EGEA Co-operative Group. Lancet 1998; 352: 985-986.

Mennie M, Gilfillan A, Brock DJ, et al. Heterozygotes for the delta F508 cystic fibrosis allele are not protected against bronchial asthma. Nat Med 1995; 1: 978-979.

Schroeder SA, Gaughan DM, Swift M. Protection against bronchial asthma by CFTR delta F508 mutation: a heterozygote advantage in cystic fibrosis. Nat Med 1995; 1: 703-705.

Lazaro C, de Cid R, Sunyer J, et al. Missense mutations in the cystic fibrosis gene in adult patients with asthma. Hum Mutat 1999; 14: 510-519.

Agarwal R, Khan A, Aggarwal AN, et al. Link between CFTR mutations and ABPA: a systematic review and meta-analysis. Mycoses 2012; 55: 357-365.

540-541.

Metersky ML. The initial evaluation of adults with bronchiectasis. Clin Chest Med 2012; 33: 219-231.

Morrissey BM. Pathogenesis of bronchiectasis. Clin Chest Med 2007; 28: 289-296.

Russell DW, Gaggar A, Solomon GM. Neutrophil Fates in Bronchiectasis and Alpha-1 Antitrypsin Deficiency. Ann Am Thorac Soc 2016; 13: Suppl. 2, S123-S129.

Murray JF, Nadel JA, Broaddus VC, et al. Murray \& Nadel's textbook of respiratory medicine. Philadelphia, Elsevier Saunders, 2016; pp. 853-876.

King PT, Freezer NJ, Holmes PW, et al. Role of CFTR mutations in adult bronchiectasis. Thorax 2004; 59: 357-358.

Bergougnoux A, Viart V, Miro J, et al. Should diffuse bronchiectasis still be considered a CFTR-related disorder? J Cyst Fibros 2015; 14: 646-653.

Bombieri C, Benetazzo M, Saccomani A, et al. Complete mutational screening of the CFTR gene in 120 patients with pulmonary disease. Hum Genet 1998; 103: 718-722.

Casals T, De-Gracia J, Gallego M, et al. Bronchiectasis in adult patients: an expression of heterozygosity for CFTR gene mutations? Clin Genet 2004; 65: 490-495.

Girodon E, Cazeneuve C, Lebargy F, et al. CFTR gene mutations in adults with disseminated bronchiectasis. Eur J Hum Genet 1997; 5: 149-155.

Bienvenu T, Sermet-Gaudelus I, Burgel PR, et al. Cystic fibrosis transmembrane conductance regulator channel dysfunction in non-cystic fibrosis bronchiectasis. Am J Respir Crit Care Med 2010; 181: 1078-1084.

Cuppens $\mathrm{H}$, Lin W, Jaspers $\mathrm{M}$, et al. Polyvariant mutant cystic fibrosis transmembrane conductance regulator genes. The polymorphic $(\mathrm{Tg}) \mathrm{m}$ locus explains the partial penetrance of the $\mathrm{T} 5$ polymorphism as a disease mutation. J Clin Invest 1998; 101: 487-496.

Button B, Picher M, Boucher RC. Differential effects of cyclic and constant stress on ATP release and mucociliary transport by human airway epithelia. J Physiol (Lond) 2007; 580(Pt. 2): 577-592.

Drummond MB, Dasenbrook EC, Pitz MW, et al. Inhaled corticosteroids in patients with stable chronic obstructive pulmonary disease: a systematic review and meta-analysis. JAMA 2008; 300: 2407-2416.

6 Tashkin DP, Celli B, Senn S, et al. A 4-year trial of tiotropium in chronic obstructive pulmonary disease. $N$ Engl J Med 2008; 359: 1543-1554.

Calverley PM, Anderson JA, Celli B, et al. Salmeterol and fluticasone propionate and survival in chronic obstructive pulmonary disease. N Engl J Med 2007; 356: 775-789.

Godtfredsen NS, Lam TH, Hansel TT, et al. COPD-related morbidity and mortality after smoking cessation: status of the evidence. Eur Respir J 2008; 32: 844-853.

Tonnesen P, Carrozzi L, Fagerstrom KO, et al. Smoking cessation in patients with respiratory diseases: a high priority, integral component of therapy. Eur Respir J 2007; 29: 390-417.

Continuous or nocturnal oxygen therapy in hypoxemic chronic obstructive lung disease: a clinical trial. Nocturnal Oxygen Therapy Trial Group. Ann Intern Med 1980; 93: 391-398.

Long term domiciliary oxygen therapy in chronic hypoxic cor pulmonale complicating chronic bronchitis and emphysema. Report of the Medical Research Council Working Party. Lancet 1981; 1: 681-686.

Zheng JP, Kang J, Huang SG, et al. Effect of carbocisteine on acute exacerbation of chronic obstructive pulmonary disease (PEACE Study): a randomised placebo-controlled study. Lancet 2008; 371: 2013-2018. 
113 Poole PJ, Black PN. Mucolytic agents for chronic bronchitis or chronic obstructive pulmonary disease. Cochrane Database Syst Rev 2006; 3: CD001287.

114 Lambert JA, Raju SV, Tang LP, et al. Cystic fibrosis transmembrane conductance regulator activation by roflumilast contributes to therapeutic benefit in chronic bronchitis. Am J Respir Cell Mol Biol 2014; 50: 549-558.

115 Rennard SI, Calverley PM, Goehring UM, et al. Reduction of exacerbations by the PDE4 inhibitor roflumilast--the importance of defining different subsets of patients with COPD. Respir Res 2011; 12: 18

116 Tyrrell J, Qian X, Freire J, et al. Roflumilast combined with adenosine increases mucosal hydration in human airway epithelial cultures after cigarette smoke exposure. Am J Physiol Lung Cell Mol Physiol 2015; 308: L1068-L1077.

117 Raju SV, Rasmussen L, Sloane PA, et al. Roflumilast reverses CFTR-mediated ion transport dysfunction in cigarette smoke-exposed mice. Respir Res 2017; 18: 173.

118 Milara J, Morell A, Ballester B, et al. Roflumilast improves corticosteroid resistance COPD bronchial epithelial cells stimulated with toll like receptor 3 agonist. Respir Res 2015; 16: 12

119 Rabe KF, Watz H, Baraldo S, et al. Anti-inflammatory effects of roflumilast in chronic obstructive pulmonary disease (ROBERT): a 16-week, randomised, placebo-controlled trial. Lancet Respir Med 2018; 6: 827-836.

120 Solomon GM, Hathorne H, Liu B, et al. Pilot evaluation of ivacaftor for chronic bronchitis. Lancet Respir Med 2016; 4: e32-e33.

121 The Topic Trial - Study to Determine the Safety and Efficacy of Ivacaftor. https:/ClinicalTrials.gov/show/ NCT03085485 Date last accessed: 1 March 2020; date last updated: 1 March 2020.

122 Clearing Lungs With ENaC Inhibition in Primary Ciliary Dyskinesia. https://ClinicalTrials.gov/show/ NCT02871778 Date last accessed: 1 March 2020; date last updated: 1 March 2020. 